\title{
E3 ubiquitin ligase Nedd4 is a key negative regulator for non-canonical inflammasome activation
}

\author{
Qingjun Liu $\mathbb{D}^{1} \cdot$ Shihui Zhang ${ }^{1} \cdot$ Zhongjie Sun $^{2} \cdot$ Xiao Guo $^{2} \cdot$ Hong Zhou $^{1}$
}

Received: 20 June 2018 / Revised: 10 February 2019 / Accepted: 11 February 2019 / Published online: 28 February 2019

(c) ADMC Associazione Differenziamento e Morte Cellulare 2019

\begin{abstract}
The non-canonical inflammasome plays important roles in endotoxic shock and pyroptosis. Murine caspase-11, corresponding to human caspase-4, is centrally located in the non-canonical inflammasome pathway, which is directly activated by cytosolic lipopolysaccharide. It has been reported that ubiquitination strictly regulates inflammatory responses. However, the role of ubiquitination in regulating the non-canonical inflammasome is little known. In this study, we show that the E3 ubiquitin ligase, Nedd4 is an important negative regulatory component of the non-canonical inflammasome pathway. Nedd4 deficiency promoted mouse death from sepsis and cell pyroptosis, resulting from non-canonical inflammasome activation. Furthermore, Nedd4 induced the K48-linked polyubiquitination and subsequent degradation of caspase-11 through the 26S proteasome. Meanwhile, caspase-11 (or caspase-4) reciprocally regulated the level of Nedd4 protein by cleavage. Thus, Nedd4 appears to have a key role in balancing the level of non-canonical inflammasome activation in response to gram-negative bacterial infection.
\end{abstract}

\section{Introduction}

Innate immunity surveys any signs of invading pathogens or tissue injury in the extracellular and intracellular space, and depends on the activity of germline-encoded pathogen recognition receptors (PRRs). The inflammasome is a large, cytoplasmic, multi-protein complex comprising a PRR, ASC (apoptosis-associated speck-like protein containing a caspase recruitment domain) (or not) and caspase-1, and plays a crucial role in this immune surveillance [1]. Once inflammasomes have formed, caspase- 1 is activated to cleave pro-

Edited by V. D'Angiolella

Qingjun Liu

qjliu2003@163.com

$\triangle$ Hong Zhou

zhouhtt1966@163.com

1 Institute of Health Service and Transfusion Medicine, Academy of Military Medical Sciences, Taiping Road 27, Haidian District, 100850 Beijing, China

2 Newish technology (Beijing) Co., Economic and Technical Development Zone Ltd., Xihuan South Road 18, 100176 Beijing, China
IL-1 $\beta$ and pro-IL-18 into active cytokines, which eventually results in the induction of an inflammatory cell death called pyroptosis [2]. Although inflammasomes activating caspase-1 have been the focus of most studies, a non-canonical inflammasome that activates caspase-11 (murine caspase-11 corresponds to human caspase-4) [3,4] in innate immunity has been highlighted in recent years for its ability to modulate NLRP3 inflammasome activation and caspase-1-independent pyroptosis. Inappropriate or excessive inflammasome activation can be detrimental to host health [5].

The non-canonical inflammasome is activated by most Gram-negative bacteria, by transfected lipopolysaccharide (LPS), as well as by the combination of cholera toxin B (CTB) and LPS in a TLR4-independent manner $[3,6]$. In fact, only the LPS moieties, penta-acylated or hexa-acylated lipid A triggers the non-canonical inflammasome pathway, whereas tetra-acylated lipid A does not [6]. Caspase-11 and human caspase 4/5 undergo oligomerization and activation upon LPS binding to their caspase activation and recruitment domains [7]. The signaling events downstream of caspase- 11 have been gradually elucidated. Gasdermin D is essential for caspase-11-dependent pyroptosis and interleukin-1 $\beta$ maturation $[8,9]$. Besides gasdermin $\mathrm{D}$, pannexin-1, and $\mathrm{P} 2 \mathrm{X} 7$ are critical targets of caspase-11, which mediate pyroptosis and endotoxic shock caused by noncanonical inflammasome activation. The physiological 
function of caspase-11 is to discriminate cytosolic from vacuolar bacteria, which is critical for surviving exposure to ubiquitous environmental pathogens [10]. However, excessive caspase-11 activation causes lethal septic shock [3, 11-13]. Although the roles and downstream mechanisms of the non-canonical inflammasome have been illuminated, the precise regulatory mechanism for its activation remains unknown.

Ubiquitination is an important post-translational protein modification and plays a crucial regulatory role in inflammatory cells $[14,15]$. Protein ubiquitination is sequentially mediated by three enzymes: the ubiquitin-activating enzyme (E1), ubiquitin-conjugating enzyme (E2), and ubiquitin ligase (E3), which controls substrate specificity [16, 17]. E3s are divided into three groups: homologous to E6-AP $\mathrm{COOH}$ terminus (HECT), really interesting new gene (RING), and U-box E3s. In the case of HECT E3s, ubiquitin is first transferred to the active cysteine of E3, and the E3 subsequently adds it to the substrate. The other E3s directly mediate transfer of ubiquitin from E2 to the substrate. The ubiquitin can be conjugated to the substrate's lysine $(\mathrm{K})$ residues through isopeptide bonds; furthermore, it is conjugated either as a single moiety, or as K48/K63linked polyubiquitin chains, or via other $\mathrm{K}$ residues of ubiquitin, with different functional consequences [15]. In general, K48-linked polyubiquitin chains target substrates to the $26 \mathrm{~S}$ proteasome or lysosome [18] for degradation, while K63-linked polyubiquitin chains are associated with protein trafficking [19].

Neural precursor cell-expressed developmentally downregulated gene 4 (Nedd4) is one of the HECT E3s, and is characterized by an N-terminal $\mathrm{C} 2$ domain, 3-4 WW repeats and a C-terminal HECT domain. It is widely expressed in mammalian tissues [20]. Nedd4 plays distinct roles in different cellular functions since it can target different substrates [21] such as phospho-AKT [22], insulinlike growth factor 1 receptor [23], and phosphatase and tensin homolog [24]. Furthermore, Nedd4 has been found to have important regulatory roles in adaptive immunity, by mediating the ubiquitination and subsequent degradation of Cbl-b to promote the conversion of naive $\mathrm{T}$ cells into activated $\mathrm{T}$ cells [25], and by ubiquitinating TRAF3 to promote CD40-mediated AKT activation in B cells [26]. Furthermore, we previously found that Nedd4 inhibits AP-1 activation and TNF-a production through targeting p38a for polyubiquitination in macrophages [27]. However, the function of Nedd4 in non-canonical inflammasome activation is still unknown.

In this study, we demonstrate that Nedd4 plays a key negative role in non-canonical inflammasome activation in vivo and in vitro. Importantly, our data show that Nedd4 directly interacts with caspase-11 and mediates K48-linked polyubiquitination of caspase-11 at multiple $\mathrm{K}$ residues and subsequent degradation through the $26 \mathrm{~S}$ proteasome. Meanwhile, we also found that caspase-11 (or caspase-4) reciprocally regulates Nedd4 by cleavage.

\section{Results}

\section{Nedd4 inhibits endotoxic shock induced by non- canonical inflammasome activation}

It was previously reported that the E3 ligase, Cbl-b specifically inhibits canonical and non-canonical NLRP3 inflammasomes by targeting NLRP3 for ubiquitination [28]. Although Nedd4 is responsible for the ubiquitination of Cbl-b and its subsequent degradation, the role of Nedd4 in non-canonical inflammasome activation is little known. Hence, we performed experiments to assess the role of Nedd4 in an endotoxic shock model that has been developed to assess the non-canonical inflammasome in vivo [6, 29, 30]. Wild-type (WT) mice primed with non-lethal doses of LPS $(400 \mu \mathrm{g} / \mathrm{kg})$ and then challenged with higher doses of LPS $(10 \mathrm{mg} / \mathrm{kg})$ rapidly succumbed, as previously reported [6, 29, 30]. Furthermore, $\mathrm{Nedd} 4^{+/-}$mice were more susceptible to endotoxic shock compared with the WT, even though they were heterozygous for the Nedd4 deficiency (Fig. 1a and Table 1) $\left(\mathrm{Nedd} 4^{-1-}\right.$ homozygous cannot be viable). The production of IL-1 $\beta$, TNF-a, and IL6 were significantly higher in Nedd $4^{+/-}$mouse sera compared with WT sera (Fig. 1b, d). To investigate the lesions in heart, liver, spleen, lung, and kidney resulting from noncanonical inflammasome activation, we performed histological analysis of these tissues. There were no apparent differences in lesions of the spleen, lung, and kidney between WT and $\mathrm{Nedd} 4^{+/-}$mice. However, there was significantly more myocarditis in $\mathrm{Nedd} 4^{+/-}$mice than in WT mice (4/5 vs. 0/5) (Fig. 1e, upper panels). Furthermore, large flaky bleeding areas and necrosis were visible in the livers of Nedd $4^{+/-}$mice compared with WT mice (4/5 vs. 0/5) (Fig. 1e, lower panels). The pathologic changes of heart and liver tissues were more serious in Nedd $4^{+/-}$mice than in WT mice. Collectively, these results indicate that Nedd4 inhibits the rapid onset of mortality and pathological changes induced by non-canonical inflammasome activation in vivo. Since Cbl-b ubiquitination and subsequent degradation is caused by Nedd4, thus, Nedd4 deficiency should keep more Cbl-b and ideally less IL-1 $\beta$ production in response to LPS challenge. However, the result was in conflict with the expected result. Thus, we speculated that the inhibition of Nedd 4 on activation of the non-canonical inflammasome was independent of the inhibitory effect of Cbl-b on NLRP3. 
A.

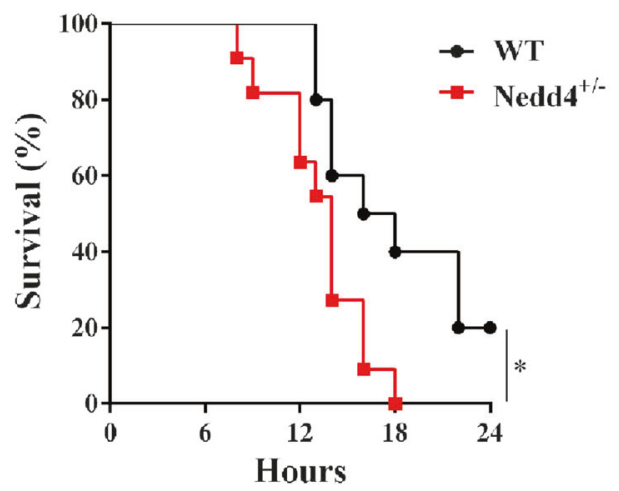

C.

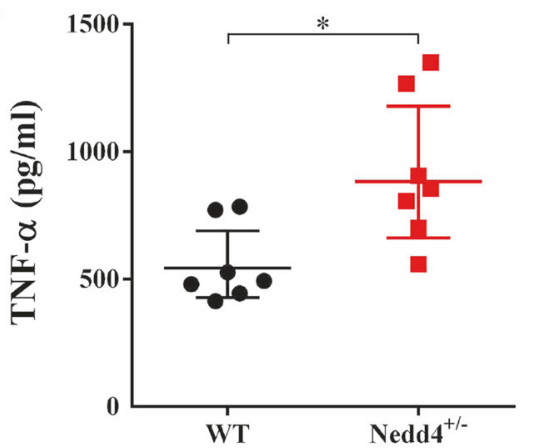

B.

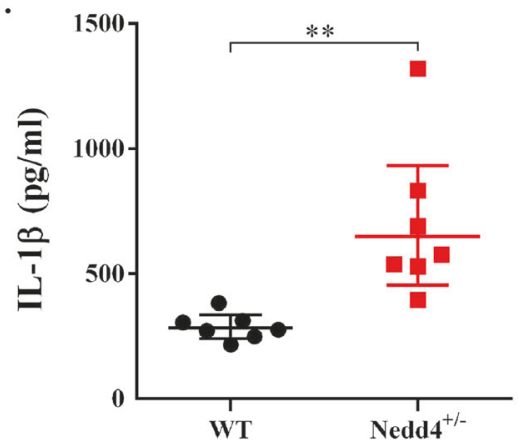

D.

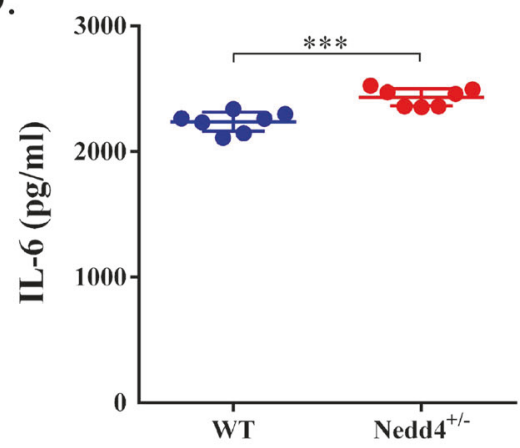

E.
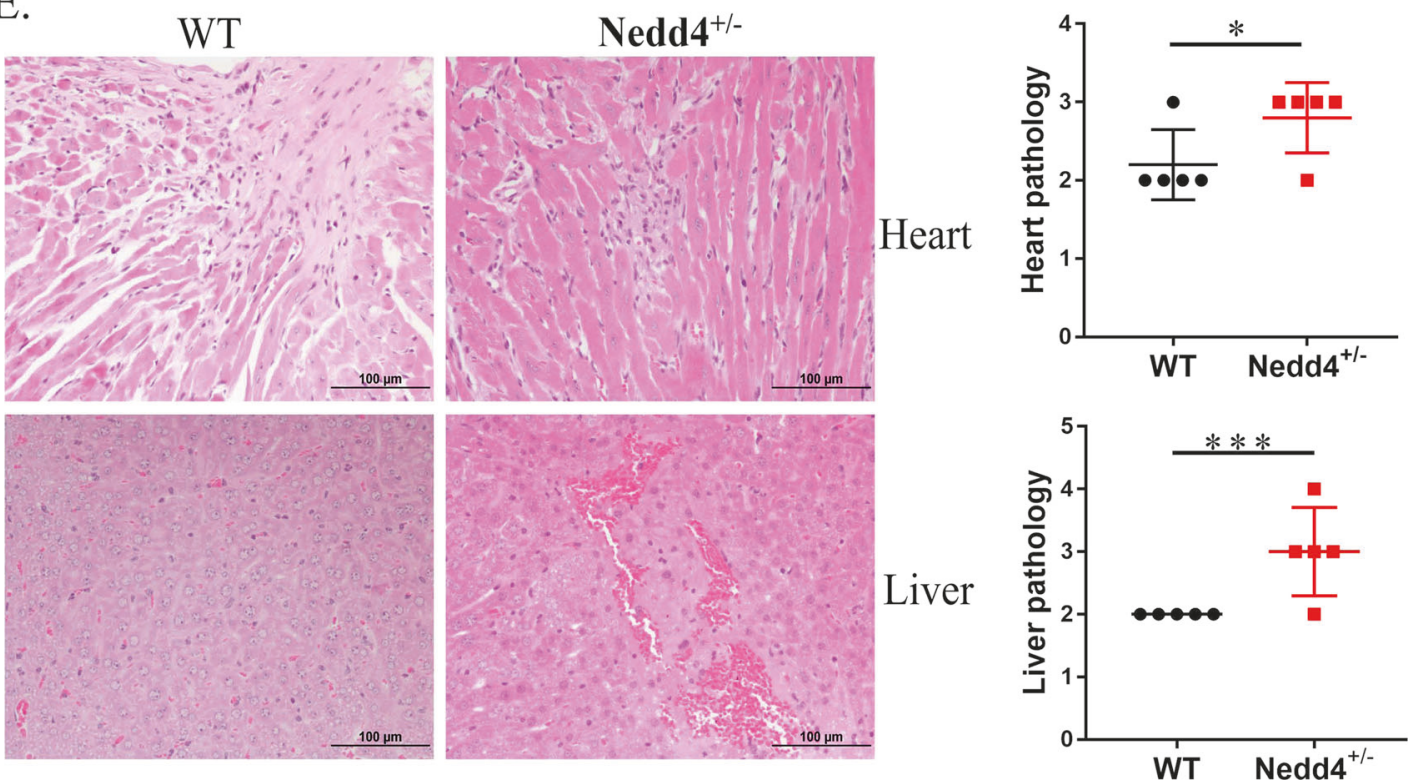

Fig. 1 Critical role of Nedd4 in endotoxic shock induced by noncanonical inflammasome activation. a Survival of mice primed with 400 $\mu \mathrm{g} / \mathrm{kg}$ E. coli O111:B4 LPS, and then challenged with $10 \mathrm{mg} / \mathrm{kg}$ E. coli O111:B4 LPS $7 \mathrm{~h}$ later. $N=10$ mice in the WT C57BL/6 $\left(\mathrm{Nedd}^{+/+}\right)$ group and $N=11$ mice in the Nedd $4^{+/-}$group $(* \mathrm{p}=0.0154, \log$-rank test). b-d. IL-1 $\beta$, TNF-a and IL- 6 levels in WT and Nedd $4^{+--}$mouse sera were measured at $8 \mathrm{~h}$ after re-challenge with LPS. $N=7$ mice in C57BL/6 (WT) and $\mathrm{Nedd}^{+/-}$groups $(* p=0.0129, * * p=0.0046$,
$* * * p=0.0005, T$-test). Error bars indicate the standard deviation (SD) of samples. e. Images of hematoxylin and eosin (H\&E) staining of the heart and liver of WT and Nedd $4^{+/-}$mice showing myocarditis in the heart and large flaky bleeding areas and necrosis in the liver. Images are representative of five independent experiments. Scale bars represent 100 $\mu \mathrm{m}$. The pathologic changes of heart and liver tissues were scored according to histopathologic grading $(* p<0.05, * * * p<0.01)$ 
Table 1 The time point of death of mice injected with LPS for noncanonical inflammasome activation

\begin{tabular}{lll}
\hline Time point (h) & WT (death) & Nedd4 $^{+/-}$(death) \\
\hline 8 & 0 & 1 \\
9 & 0 & 1 \\
12 & 0 & 2 \\
13 & 2 & 1 \\
14 & 2 & 3 \\
16 & 1 & 2 \\
18 & 1 & 1 \\
21 & 2 & 0 \\
Total (death) & 8 & 11 \\
\hline
\end{tabular}

\section{Nedd4 inhibits pyroptosis following caspase-11 activation by intracellular LPS}

Caspase-11 rather than caspase-1, is required for noncanonical inflammasome-triggered pyroptosis [3]. Nedd4 ${ }^{-/-}$ iBMDM cells established by the CRISPR/Cas9 method [27] were selected to assess the role of Nedd4 in the pyroptosis induced by activation of non-canonical inflammasomes. The pyroptosis induced by stimulation of LPS-primed iBMDM cells with CTB and LPS [3, 29] was assessed. The pyroptosis of Nedd4 ${ }^{-/-}$iBMDM cells was greater than that of WT iBMDM cells (Fig. 2a). To confirm the role of Nedd4 in the pyroptosis induced by non-canonical inflammasomes, the pyroptosis of Nedd $4^{-1-}$ iBMDM cells caused by electrotransfection of LPS [7] was also determined. As expected, the pyroptosis induced in LPS-electrotransfected Nedd ${ }^{-1-}$ iBMDM cells was significantly enhanced in comparison with WT controls (Fig. 2b). Thus, Nedd4 inhibits the pyroptosis caused by non-canonical inflammasome activation.

Since Nedd4 is one of the E3 ubiquitin ligases, we speculated that the inhibitory effect of Nedd4 on pyroptosis caused by non-canonical inflammasome activation might be mediated by its ligase activity. To test the hypothesis, we assessed the pyroptosis of $293 \mathrm{~T}$ cells co-transfected caspase11 and Nedd4 or Nedd4C744E, the E3 ligase activity of which is lost. Nedd4 indeed inhibited the pyroptosis induced by caspase-11 (Fig. 2c, d). Furthermore, Nedd4C744E (Nedd4 mutant, which lacks E3 ligase activity) induced a weaker inhibitory effect. Thus, Nedd4 inhibits the pyroptosis induced by caspase-11 activation through its E3 ubiquitin ligase activity.

\section{Nedd4 controls the protein level of caspase-11}

Since caspase-11 is centrally located in the non-canonical inflammasome pathway, it might be one of the regulatory targets of Nedd4. To determine whether Nedd4 is responsible for regulating the level of caspase-11 protein, we evaluated caspase-11 protein levels in Nedd4-deficient iBMDM cells. When Nedd4 expression was knocked down with a short hairpin RNA (shRNA), caspase-11 protein levels following LPS treatment were significantly increased compared with the controls (Fig. 3a). To confirm that Nedd4-silencing was not due to an off-target effect of RNA interference, we also evaluated the levels of caspase-11 protein in Nedd4 $4^{-1-}$ iBMDM cells. The levels of caspase-11 in these cells at different time points following LPS stimulation were also markedly increased compared with the controls (Fig. 3b). From Fig. 3a we could see that caspase-11 expression in shRNA control clones was very lower than that in WT iBMDM cells. The caspase-11 expression induced by LPS for $1 \mathrm{~h}$ in shRNA control clones was comparable with that in normal iBMDM with no LPS treatment (Fig. 3b), thus, we speculated that the control shRNA affected the caspase-11 expression. To avoid the interference caused by caspase11 expression of the clones, we used the wild types iBMDM as controls in Fig. 3b. Furthermore, the caspase11 expression was also slower in response to LPS treatment in normal iBMDM, thus we changed the LPS treatment length to $3 \mathrm{~h}$ and $6 \mathrm{~h}$.

p38 $\alpha$ regulates the expression of caspase- 11 mRNA [31], and is polyubiquitinated by Nedd4. Thus, Nedd4 might indirectly inhibit caspase-11 mRNA expression by ubiquitinating $\mathrm{p} 38 \alpha$. To discriminate between the effects of Nedd 4 on caspase-11 mRNA and protein expression, we added a specific inhibitor of p38 kinase (SB203580) into the cell culture medium, to inhibit any effect of $\mathrm{p} 38 \alpha$ on caspase-11 mRNA expression. The different exposure of Western blots likely accounts for the seeming inconsistency between the level of caspase-11 of WT at $6 \mathrm{~h}$ in Fig. $3 \mathrm{~b}$ and that in Fig. 3e. p38 is not the only positive transcription regulator of caspase-11 expression, for example, NF- $\mathrm{\kappa B}$ is another positive transcription regulator of caspase-11 expression. Thus, it is not surprising that caspase-11 is still be expressed in iBMDM cells treated with the p38 inhibitor SB203580. Following inhibition of p38 kinase, there was still more caspase-11 protein in Nedd $4^{-l-}$ compared with WT iBMDM cells (Fig. 3e). This result shows that Nedd4 directly controls caspase-11 protein levels, aside from its effect on p38 $\alpha$ upstream of caspase-11 expression.

To further investigate whether the E3 ubiquitin ligase activity of Nedd4 is responsible for regulating casapse-11 protein levels, we assessed the effects of Nedd4 and Nedd4C744E on caspase-11 expression in HEK293T cells. By transfecting increasing amounts of Nedd4, we found that it dose-dependently decreased caspase-11 protein levels (Fig. 3c). Furthermore, Nedd4C744E, which lacks E3 ligase 
A.

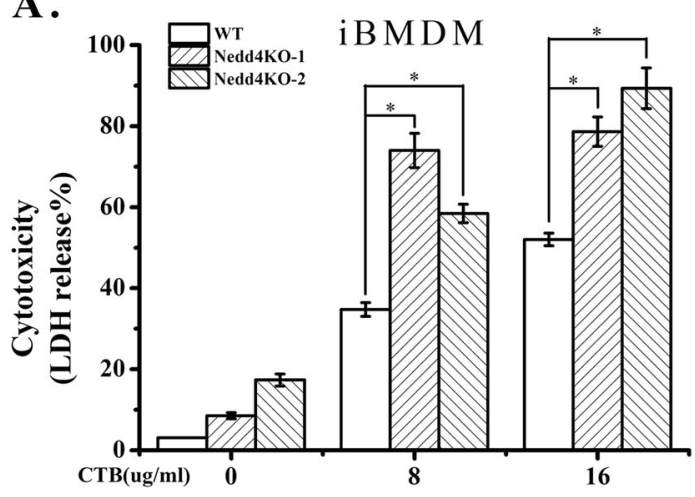

C.

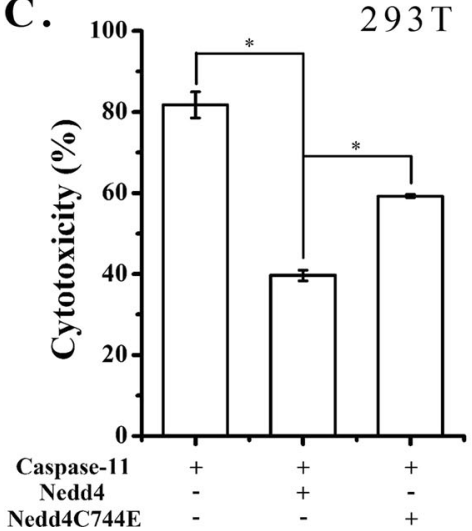

Fig. 2 Nedd4 inhibits pyroptosis induced by non-canonical inflammasome activation. a Cytotoxicity was examined in WT and $\mathrm{Nedd} 4^{-/-}$ iBMDM cells. The iBMDM cells were primed with $500 \mathrm{ng} / \mathrm{ml}$ LPS for 4 $\mathrm{h}$, then $2 \mu \mathrm{g} / \mathrm{ml}$ LPS plus $8 \mu \mathrm{g} / \mathrm{ml}$ or $16 \mu \mathrm{g} / \mathrm{ml} \mathrm{CTB}$ for $16 \mathrm{~h}$. Nedd $4 \mathrm{KO}-1$ and Nedd4KO-1 are Nedd4 ${ }^{-1-}$ iBMDM cell lines. b The cytotoxicity induced in WT and Nedd $4^{-1-}$ iBMDM cells by electrotransfected LPS was examined. c Nedd4 inhibits pyroptosis via its E3 ubiquitin ligase

activity, did not similarly decrease caspase- 11 protein levels (Fig. 3d).

To further the effect of Nedd 4 on the caspase-11 protein in vivo, we detected the caspase-11 protein levels of the liver of WT and Nedd $4^{+/-}$mice treated with LPS or without LPS using immunohistochemistry. There were still more caspase-11 protein in the livers of Nedd $4^{+/-}$mice compared with WT mice treated with LPS (Fig. 3f, bottom left) or without LPS (Fig. 3f, upper left). Furthermore, there were more aggregated caspase- 11 in the livers of Nedd $4^{+/-}$mice treated with LPS (Fig. 3f, bottom left). The quantitative results by Image Pro Plus (Fig. 3F, right) further showed that LPS induced caspase-11 expression and Nedd4 deficiency led to higher caspase-11 levels.

Collectively, these results indicate that Nedd4 is an important regulator of caspase-11 protein levels.
B.

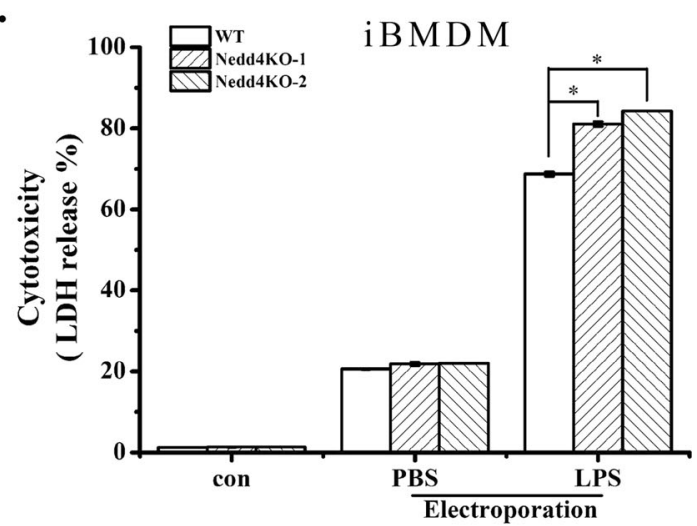

D.

\section{$293 \mathrm{~T}$}

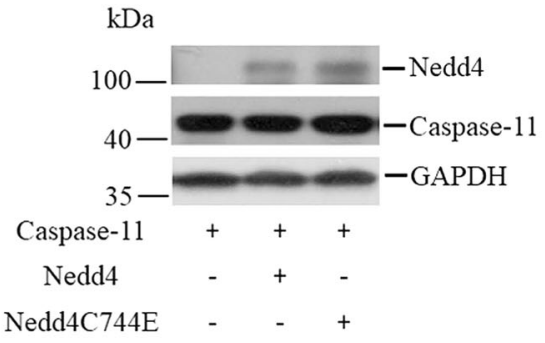

activity. HEK293T cells were co-transfected with caspase-11-Flag and Nedd4 or Nedd4C744E, and were then electrotransfected with LPS. The resulting pyroptosis was assessed. For $\mathbf{a}-\mathbf{c}$, data are representative of at least three experiments. Graphs show mean \pm SD of triplicate cultures, $* p<0.05$. d The Nedd4, Nedd4C744E and caspase- 11 expressions in 293T cells (Fig. 3C) were confirmed by Western blot using anti-Nedd4 and anti-Flag antibody, respectively

\section{Nedd4 interacts with endogenous and exogenous caspase-11}

As described above, Nedd4 regulates the protein levels of caspase-11. Thus, we hypothesized that caspase-11 might be a direct substrate of Nedd4 and that caspase-11 ubiquitination by Nedd 4 might inhibit non-canonical inflammasome activation. To test this hypothesis, we used co-immunoprecipitation experiments. We found that Nedd4 physically interacted with caspase- 11 in iBMDM cells treated with LPS (Fig. 4a). To confirm the interaction between Nedd4 and caspase-11, we co-transfected vectors expressing Nedd4 and caspase-11 into HEK293T cells. The results of immunoprecipitation with anti-Nedd4 and antiFlag antibodies showed that Nedd4 could interact with caspase-11 (Fig. 4b, c). 
A.

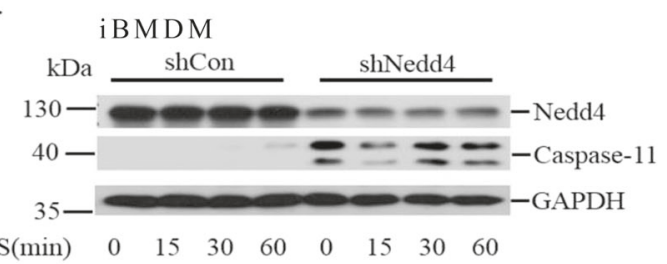

C.

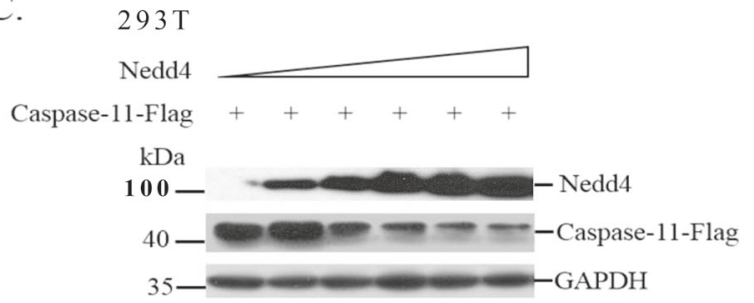

E.

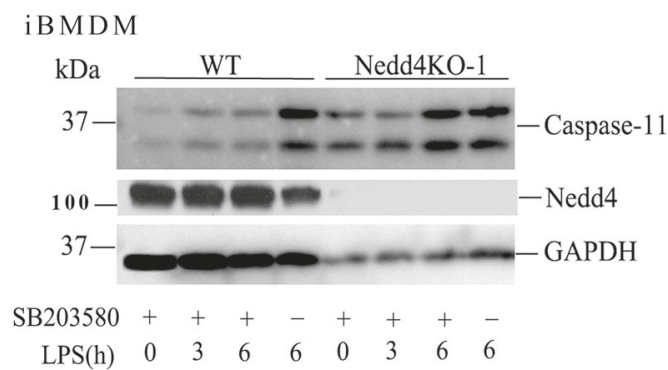

F.

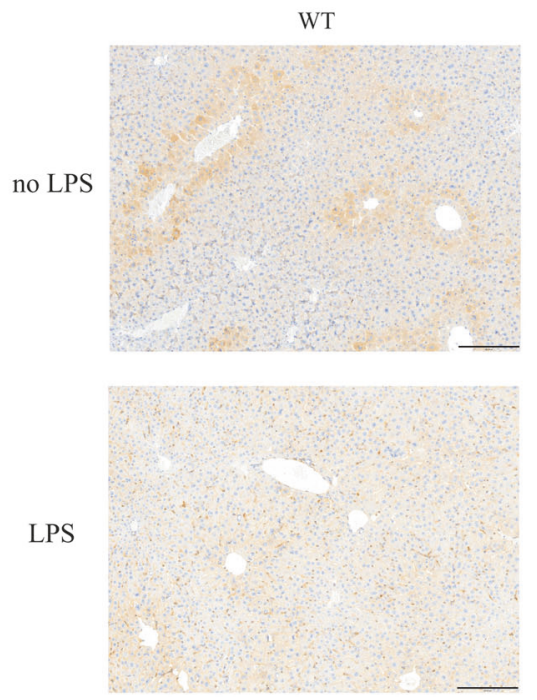

B.

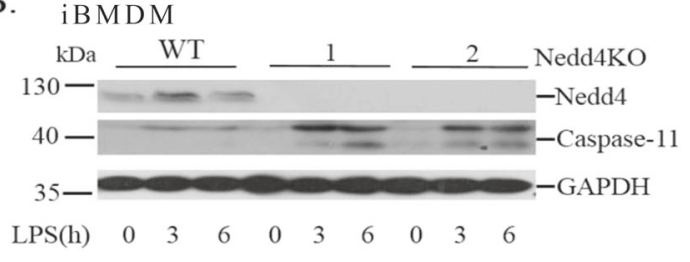

D. $293 \mathrm{~T}$
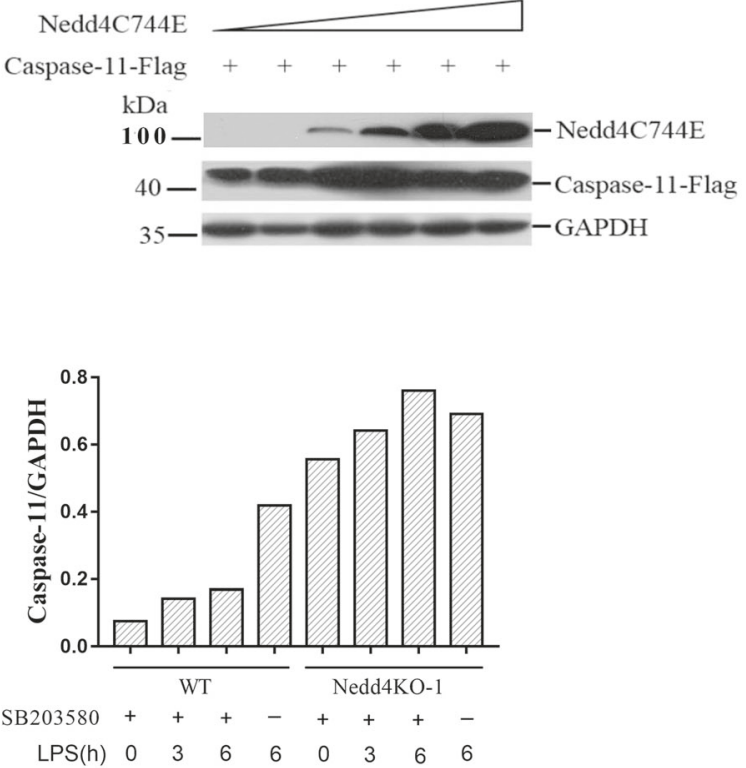

Nedd4 ubiquitinates caspase-11 with K48-linked polyubiquitin chains; ubiquitinated caspase-11 then degrades through the $26 \mathrm{~S}$ proteasome

Since Nedd4 is an E3 ubiquitin ligase, we speculated that Nedd4 might be responsible for the polyubiquitination of caspase-11. To test this hypothesis, we evaluated caspase-
Nedd $4^{+/-}$

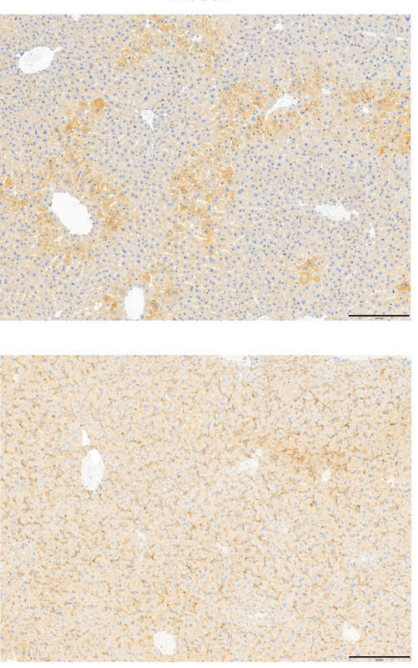

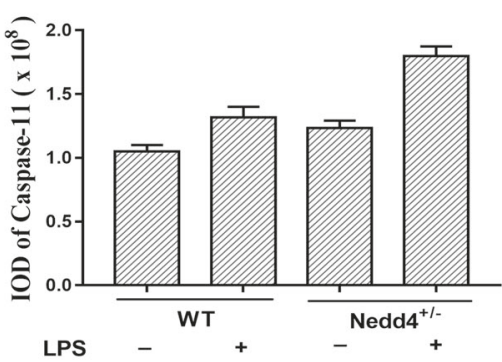

11 ubiquitination in vitro by co-transfecting caspase-11Flag, ubiquitin-polyhistidine, and hemagglutinin (HA) Nedd4 into $293 \mathrm{~T}$ cells. Meanwhile, we similarly cotransfected Nedd4C744E with the other plasmids to confirm the role of Nedd 4 in the ubiquitination of caspase- 11 . The immunoblotting results (Fig. 5a) showed that Nedd4 mediated the polyubiquitination of caspase- 11 . 
Fig. 3 Nedd 4 controls the protein level of caspase-11. a-b Nedd4 deficiency increases caspase-11 protein levels. a Caspase-11 protein levels were increased in Nedd4shRNA-treated iBMDM cells compared with controls, following stimulation with LPS for 0-60 min (times shown below lanes). GAPDH is used as an internal loading control in the experiments (including below experiments). b Caspase11 protein levels were increased in two $\mathrm{Nedd}^{-1-}$ iBMDM cell lines stimulated with LPS for 0-6h (times below lanes). c, d Nedd4mediated degradation of caspase-11 depends on its E3 ubiquitin ligase activity. Immunoblot analysis of lysates from HEK293 cells cotransfected with equivalent amounts of caspase-11-Flag plus increasing amounts of Nedd4 (C) or Nedd4C744E (D). Nedd4 and Nedd4 (C744E), and caspase-11 were immunostained with anti-Nedd4 and anti-Flag antiboy, respectively. e Caspase-11 protein levels were still increased in Nedd $4^{-1-}$ iBMDM cells stimulated with LPS for $0-6 \mathrm{~h}$ (times below lanes) in the presence of the p38 inhibitor, SB203580. The iBMDM cells were pretreated with $20 \mu \mathrm{M}$ SB203580 for $1 \mathrm{~h}$ before LPS treatment. The caspase-11 level in each lane was normalized to GAPDH, respectively (right). f Images of caspase-11 staining of the livers of WT and $\mathrm{Nedd}^{+/-}$mice showing more caspase-11 in the livers of Nedd $4^{+-}$mice compared with WT mice treated with LPS (down) or without LPS (up). Images are representative of five independent experiments. Scale bars represent 200 $\mu \mathrm{m}$. Each group contains 5 mice. The caspase- 11 expressions in livers were quantitatively analyzed by Image Pro Plus software

Furthermore, the Nedd4C744E, as expected, could not ubiquitinate caspase-11. To confirm the type of caspase-11 polyubiquitination induced by Nedd4, we transfected caspase-11-Flag, K48-ubiquitin-HA or K63-ubquitin-HA, and HA-Nedd4 into HEK293T cells. The results showed that Nedd4 induced K48-linked, but not K63-linked, polyubiquitination of caspase-11 (Fig. 5b). Thus, Nedd4 is responsible for K48-linked polyubiquitination of caspase11. There has not been unified conclusion how many ubiquitins are added to substrates by E3 ubiquitin ligases till now. The ubiquitin numbers of polyubiquitinated caspase11 by nedd 4 might be different, which might confer different characteristic of degradation. The $100-170 \mathrm{kDa}$ and $55-70 \mathrm{kDa}$ bands might be more stable and the $35-40 \mathrm{kDa}$ band might be result from degradation of polyubiquitinated caspase-11 (Fig. 5a). The discrepancy of polyubiquitination bands of caspase-11 between Fig. 5a, b which might be result from different acquiring ability to get target protein of Ni-NTA and anti-Flag antibody.

In general, K48-linked polyubiquitination predominantly targets proteins for proteasomal or lysosomal degradation. To determine the pathway of caspase-11 degradation, we used inhibitors of the proteasomal (MG132) or lysosomal (chloroquine) pathways to assess the degradation route of caspase-11 in iBMDM cells. The protein levels of caspase11 in iBMDM cells treated with MG132 were significantly increased compared with controls (Fig. 5c). However, the protein levels of caspase-11 in cells treated with chloroquine were not increased. These results demonstrated that the caspase- 11 ubiquitinated by Nedd 4 is degraded through the $26 \mathrm{~S}$ proteasome.

\section{Multiple $\mathrm{K}$ residues of caspase-11 are polyubiquitinated by Nedd4}

To determine the sites of Nedd4-mediated polyubiquitination of caspase-11, caspase-11-Flag, Nedd4-HA, and ubiquitin-HA were co-expressed in HEK293T cells to force ubiquitination. Nedd4C744E-HA was similarly coexpressed with caspase-11-Flag and ubiquitin-HA to act as a control. After immunoprecipitating caspase-11 with antiFlag purification beads, the samples were separated by SDSPAGE and visualized by Coomassie blue staining (Fig. 6a). To analyze ubiquitinated caspase- 11 by mass spectrometry (MS), three gel regions (Fig. 6a) which might contain ubiquitin-modified protein were excised, digested with trypsin, and analyzed by liquid chromatography-MS. A database search of the MS/MS spectra revealed that caspase11 was the predominant protein identified in the samples. Representative spectra demonstrated that the K62, K63, and K133 residues of caspase-11 in gel region 1\# (Fig. 6b), and the K62, K63, K159, K172, and K312 residues of caspase11 in gel region 2\# (Fig. 6c) were modified with ubiquitin. There was no ubiquitinated lysine in caspase- 11 from the control immunoprecipitate, although there was a questionable ubiquitinated band (Fig. 6a). Overall, the results show that Nedd4 ubiquitinates caspase-11 at multiple $\mathrm{K}$ residues.

\section{Nedd4 can be cleaved by caspase- 11 and caspase- 4}

Nedd4 can be cleaved by caspase-1, 3, 6, and 7 [32], however, the effect of caspase-11 on Nedd4 is unknown. From an analysis of the Nedd4 protein sequence, we found that it includes a recognition motif for caspase-11 (or human caspase-4; the motifs are ${ }_{561}$ WIEFDG $_{566}$ in murine Nedd4 and ${ }_{993}$ WIEFDG $_{998}$ in human) [33]. Thus, we hypothesized that Nedd4 might also be cleaved by caspase- 11 . The increased caspase-11 induced by LPS decreased the levels of Nedd4 protein in iBMDM cells (Fig. 7a), which was in consistence with the previous results (Fig. 3e, the 4th lane). To further test our hypothesis, we co-transfected mouse Nedd 4 with increasing amounts of caspase- 11 or its catalytically inactive mutant, caspase-11C254A, into HEK293T cells. As expected, Nedd4 was cleaved by caspase-11 in a dose-dependent manner (Fig. 7b). Furthermore, Nedd4 was unaffected by increasing amounts of caspase-11C254A (Fig. 7c). To determine whether caspase4 can cleave Nedd 4 in humans, we co-transfected human Nedd 4 with increasing amounts of caspase- 4 or its catalytically inactive mutant, caspase-4C258A, into HEK293T cells. We similarly observed that the Nedd4 protein was gradually decreased with increasing amounts of caspase-4 (Fig. 7d) and was not affected by caspase4C258A (Fig. 7e). Thus, caspase-11 and caspase-4 


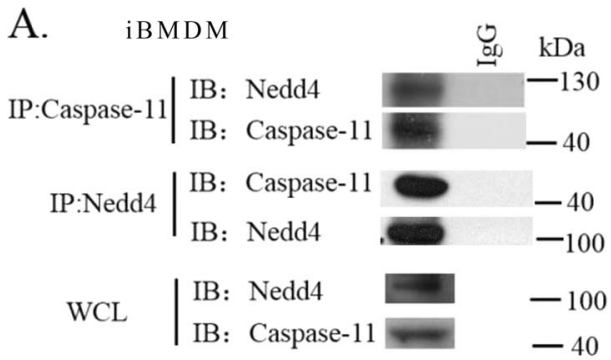

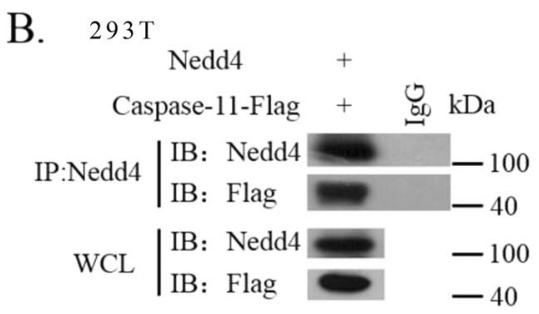

Fig. 4 Nedd4 interacts with endogenous and exogenous caspase-11. a Endogenous caspase-11. iBMDM cells were incubated with LPS for $4 \mathrm{~h}$, lysed and immunoprecipitated with anti-caspase- 11 or anti-Nedd 4 antibody. Both the immunoprecipitates (IP) and whole cell lysates (WCL) were subjected to gel electrophoresis and immunostaining with an anti-Nedd4 or anti-caspase-11 antibody. b, c Exogenous caspase-

specifically cleave Nedd4 in non-canonical inflammasome activation.

\section{Discussion}

The non-canonical inflammasome formed by caspase- 11 in mice is a developing area of interest in the inflammasome field [34]. However, the regulation of its activation by ubiquitination is poorly understood. In this study, we show that the E3 ubiquitin ligase, Nedd4 is an important negative regulator of non-canonical inflammasome activation through its K48-linked polyubiquitination of caspase-11. Furthermore, we report that caspase-11 (or human caspase-4) reciprocally regulates the level of Nedd4 protein by cleavage.

Polyubiquitination is a post-translational modification that influences the activity and function of innate sensors and downstream signaling molecules through the covalent linkage of ubiquitin to lysine residues in target proteins [35]. Recent studies have reported that the non-canonical inflammasome plays very important roles in innate immunity. Thus, it is reasonable to suppose that the polyubiquitination of inflammasome components precisely regulates non-canonical inflammasome activity in response to different levels of infection and danger signals.

Nedd4 is well-known to play important roles in development and physiological growth. Mice deficient in Nedd4 $\left(\mathrm{Nedd}^{-l-}\right.$ mice) die at birth, with delayed embryonic development and reduced growth and body weight [23]. Moreover, Nedd4 also regulates the functions of immune

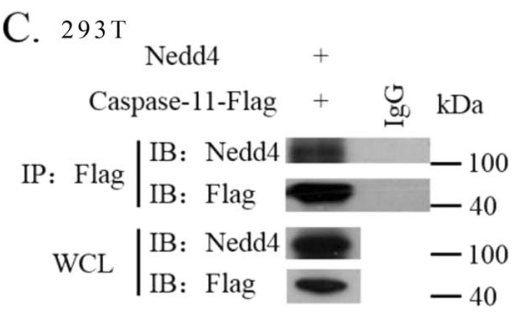

11. HEK293T cells co-transfected with Nedd4 and caspase-11 vectors were lysed and immunoprecipitated with anti-Nedd4 (B) or anti-FLAG (C) antibodies. IgG bands indicated IP results of un-transfected HEK293T cells using IP antibodies. Both the IP and WCL were subjected to gel electrophoresis and immunostaining with anti-Nedd4 or anti-Flag antibody

cells, including T cells [25], B cells [26] and macrophages [27]. However, the role of Nedd4 in regulating noncanonical inflammasomes has not previously been described. We found that heterozygous $\mathrm{Nedd} 4^{+/-}$mice show higher mortality, greater release of inflammatory cytokines and pathological changes (Fig. 1), demonstrating that Nedd4 is a very important negative regulator of noncanonical inflammasome activation. Caspase-11 directly mediates cell pyroptosis and mouse mortality [36, 37], and promotes NLRP3 inflammasome activation to indirectly enhance the processing of pro-IL-1 $\beta$ [3]. Thus, the increased IL-1 $\beta$ in $\mathrm{Nedd}^{+/-}$mouse serum might result from an indirect role of caspase-11 activation in the production of IL- $1 \beta$. The increased TNF-a in Nedd $4^{+-}$mouse serum might result from the lost control of p38a by Nedd4 [27]. The detailed mechanism of the increased IL- 6 in Nedd $4^{+/-}$ mouse serum needs to be investigated.

There were multiple $\mathrm{K}$ residues of caspase- 11 that can be ubiquitinated by Nedd4, which could not be predicted by Ubipredit online software. The K62 and K63 of caspase-11 are important sites responsible for LPS binding and subsequent caspase-11 oligomerization [7]. Interestingly, both sites are also ubiquitinated sites by Nedd4 (Fig. 6). The effect of ubiquitination of $\mathrm{K} 62$ and $\mathrm{K} 63$ by Nedd 4 on caspase-11 binding LPS and its subsequent oligomerization needs to be investigated.

There are very complicated regulation mechanisms among LPS, caspase-11 and Nedd4. LPS induces caspase11 expression and binds to caspase- 11 , and subsequently makes caspase- 11 oligomerize and activate. Here, we found 
A.

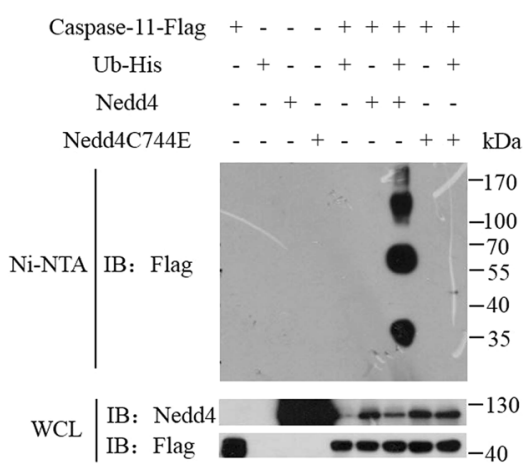

B.

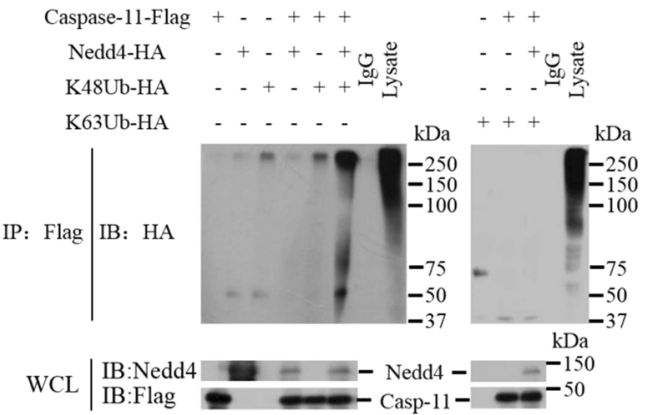

C. ${ }_{k D a}$

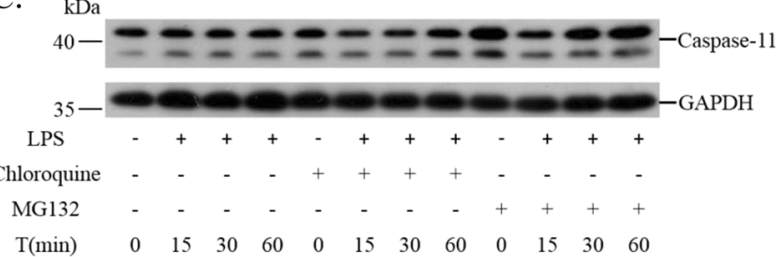

Fig. 5 Caspase-11 is K48-polyubiquitinated by Nedd 4 and its subsequent degradation is through the $26 \mathrm{~S}$ proteasome. a Caspase- 11 is polyubiquitinated by Nedd4. Immunoblot analysis of the Ni-NTAbinding fraction of lysates from HEK293T cells co-transfected with various vectors (shown above the lanes), stained with an anti-Flag antibody. For whole cell lysates (WCL), caspase-11 was stained with anti-Flag, and Nedd4 was stained with anti-Nedd4. b Caspase-11 is K48-polyubiquitinated by Nedd4. Immunoblot analysis of anti-Flag immunoprecipitates of lysates from HEK293T cells co-transfected with various vectors (shown above lanes), stained with an anti-HA antibody. For whole cell lysates (WCL), caspase-11 was stained with anti-Flag, and Nedd4 was stained with anti-Nedd4. For A-B, the results shown are representative of at least three independent experiments. c Ubiquitinated caspase- 11 is degraded through the $26 \mathrm{~S}$ proteasome pathway. Immunoblot analysis of caspase-11 in WT iBMDM cells stimulated for 0-60 min (times shown below lanes) with LPS and chloroquine or MG132. The results shown are representative of at least three independent experiments

Nedd4 interacts caspase-11 not being required for LPS (Fig. $4 \mathrm{~b}, \mathrm{c}$ ) and subsequently polyubiquitinates caspase-11, demonstrating that regulation of caspase- 11 by Nedd 4 is before activation of caspase-11 by LPS. However, the detailed mutual regulation among LPS, caspase-11 and Nedd4 need to be further investigated.
To ensure the most favorable outcome for the host, there should be a coordinated balance between the positive and negative regulation of PRR-initiated innate inflammatory responses [35, 38]. In addition to the expected finding that Nedd4 negatively controls non-canonical inflammasome activation, we surprisingly found that caspase-11 also reciprocally regulates Nedd 4 protein levels by cleavage. Nedd4 protein levels were decreased when caspase- 11 or caspase-4 were over-expressed, but the inactive mutants, caspase-11C254A and caspase-4C258A could not similarly decrease Nedd 4 protein levels. Thus, the decreased Nedd 4 protein levels appeared to result both directly from the cleavage of Nedd 4 by caspase- 11 , and indirectly from the cleavage of Nedd4 by caspase-1 [32] following its activation by caspase-11 (Fig. 7f). Taken together, our data point to an important negative regulatory mechanism in noncanonical inflammasome activation. They suggest that, in addition to its previously described function in p38a activation upstream of caspase-11 expression, the E3 ubiquitin ligase, Nedd4 also controls non-canonical inflammasome activation through the polyubiquitination and subsequent degradation of caspase-11 (Fig. 7e). Our results also suggest a reciprocal regulatory role of caspase- 11 , which mediates Nedd4 cleavage.

\section{Methods}

\section{Mice}

Nedd $4^{+/-}$mice have been previously described and were housed at the Animal Facility of the Academy of Military Medical Sciences under specific pathogen-free conditions. The mice were bred by mating between Nedd $4^{+/}$ mice and wild-type C57BL/6J mice. The genotypes of offspring were identified by the polymerase chain reaction method, and WT offspring were used as controls. Eight-week-old mice were used for LPS treatment, as previously described [30]. In brief, mice were primed with E. coli O111:B4 LPS $(400 \mathrm{mg} / \mathrm{kg})$ delivered by intraperitoneal (i.p.) injection. After $7 \mathrm{~h}$, they were challenged with $10 \mathrm{mg} / \mathrm{kg}$ LPS (E. coli O111:B4) i.p. All animal experiments were performed in accordance with the National Institute of Health Guide for the Care and Use of Laboratory Animals, and with the approval of the Scientific Investigation Board of the Academy of Military Medical Sciences.

\section{Reagents and materials}

The following commercial antibodies were used: horseradish peroxidase (HRP)-conjugated anti-mouse immunoglobulin $\mathrm{G}$ (IgG) (sc2005), HRP-conjugated anti-rabbit 


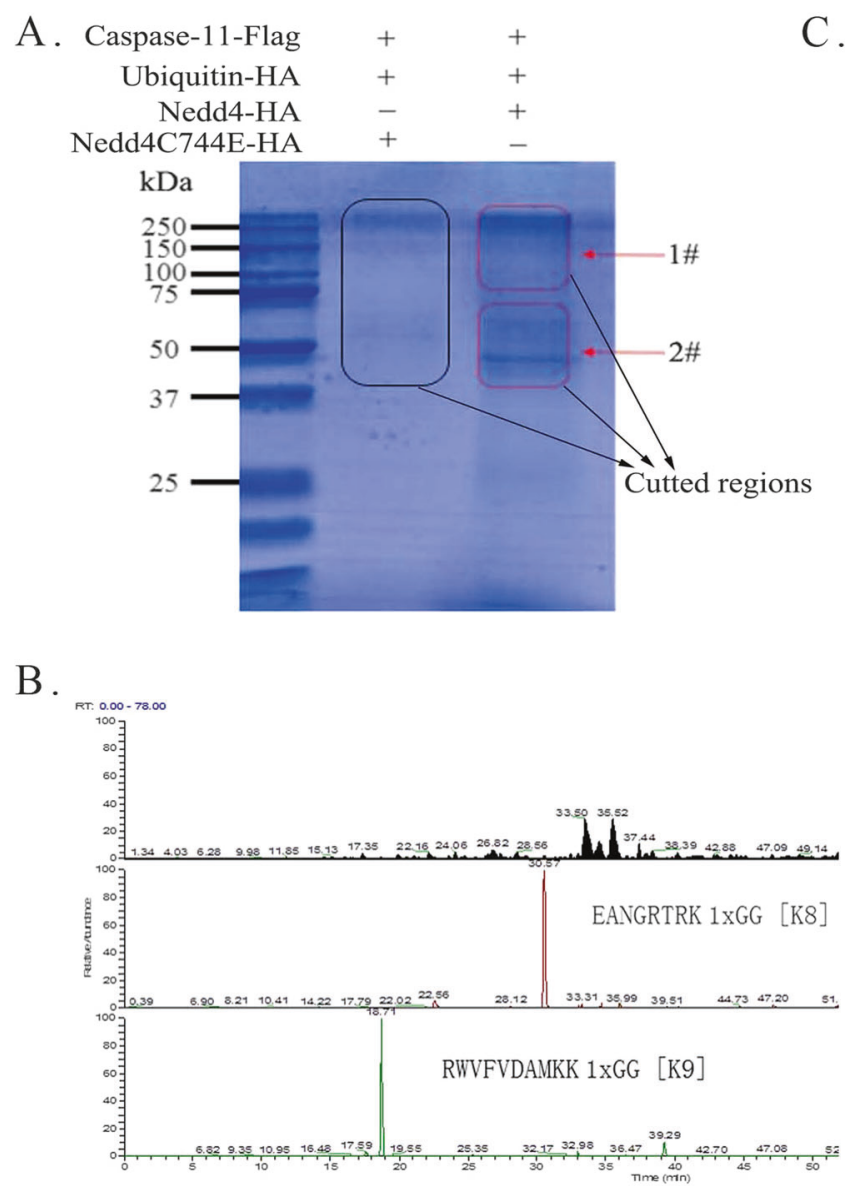

Fig. 6 Analysis of caspase 11 polyubiquitination sites. a A Coomassie blue-stained gel showing polyubiquitinated caspase-11 bands. HEK293T cells co-transfected with caspase-11-Flag, Nedd4-HA or Nedd4C744E-HA and ubiquitin-HA (shown above the lanes) were lysed, immunoprecipitated with anti-Flag purification beads, gelseparated, and stained with Coomassie blue. The square areas indicate

IgG (sc2004), HRP-conjugated anti-rat IgG (sc2006), and anti-NEDD4-1 (H-135) rabbit polyclonal IgG (sc25508) were from Santa Cruz (CA, USA). The HRP-conjugated VeriBlot for IP secondary antibody (ab131366) was from Abcam. Anti-caspase-11 (17D9) rat monoclonal antibody (\#14340) was from CST, anti-GAPDH rabbit IgG was from Proteintech, rabbit anti-HA antibody (H6908-2ML) was from Sigma, and Flag-Tag mouse monoclonal antibody (KM8002) was from SanJian (Tianjin, China). Anti-flag M2 affinity Gel (A2220), 011:B4 LPS, MG132 (C2211) and chloroquine (C6628) were obtained from Sigma. Protein A/G-Sepharose was purchased from Santa Cruz. A protease inhibitor cocktail (539134) was purchased from Calbiochem. SB202580 (HY-10256) was purchased from MCE. A murine TNF- $\alpha$ (Mini EDK) enzyme-linked immunosorbent assay (ELISA) kit (900-M54) was from PeproTECH. Murine IL-1 $\beta$ and IL-6 ELISA kits were from Biolegend. A Cytotoxicity Detection Kit (LDH) was purchased from Roche (Ref: 11644793001). Ni-NTA superflow sepharose

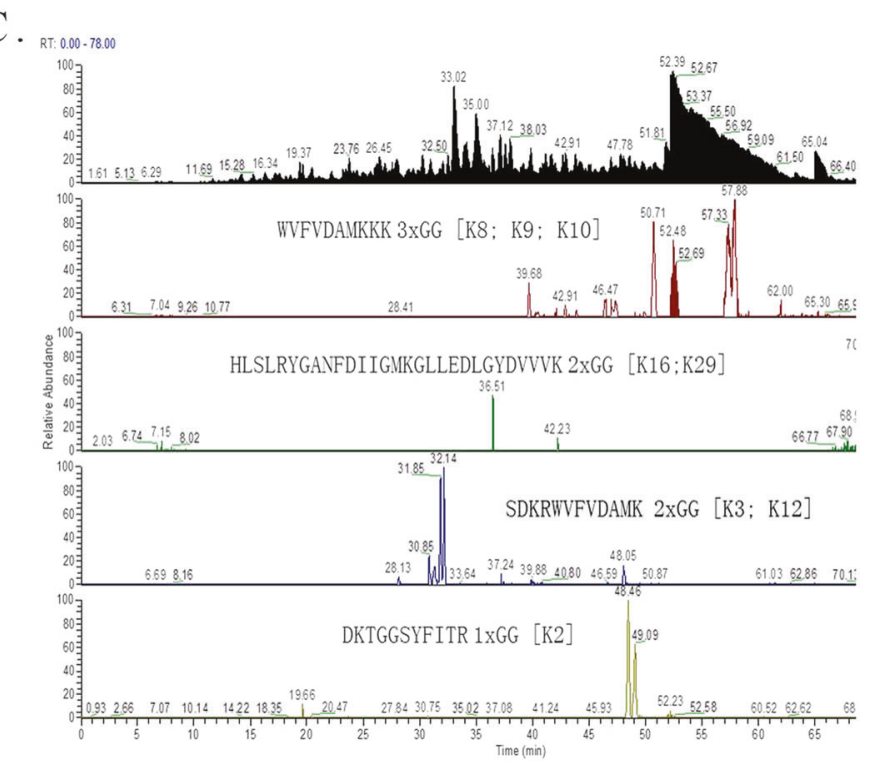

the regions excised for MS. b, c Representative MS/MS spectra of ubiquitinated caspase-11 peptides. Protein samples were recovered from the gel, enzyme digested, and subjected to MS. Two caspase-11 peptides containing ubiquitinated lysines were identified in excised region $1 \#(\mathbf{b})$, and four were identified in excised region $2 \#$ (c)

was from CMCTag (P3403). Sequencing-grade trypsin was purchased from Promega $(0.5 \mathrm{mg} / \mathrm{ml})$.

\section{Cell culture and treatment}

Human HEK293T cells were obtained from the American Type Culture Collection (Manassas, VA). C57BL/6 mousederived iBMDM cells were a gift from Professor Feng Shao (NIBS). The Nedd4 shRNA iBMDM cell line and Nedd4 knockout iBMDM cell lines (Nedd4KO-1 and Nedd4KO-2) have been previously described [27]. The cells were grown in Dulbecco's modified Eagle's medium containing $10 \%$ fetal bovine serum (complement-inactivated at $56{ }^{\circ} \mathrm{C}$ ) and $1 \%(\mathrm{v} / \mathrm{v})$ penicillin-streptomycin solution. For caspase-11 expression, iBMDM cells were incubated with $500 \mathrm{ng} / \mathrm{ml}$ LPS for the indicated times. LPS transfection was according to a previously described method [7]. In brief, $1 \times 10^{6}$ cells were transfected with $1 \mu \mathrm{g}$ LPS using the Neon Transfection System (Life technologies), following the manufacturer's instructions. 
A.

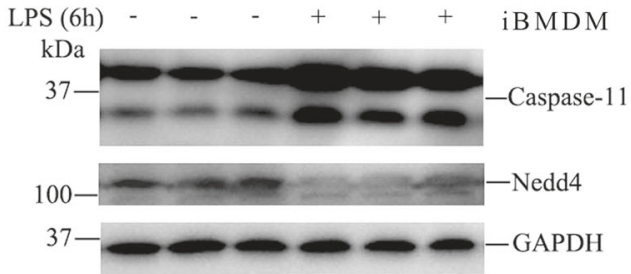

B.
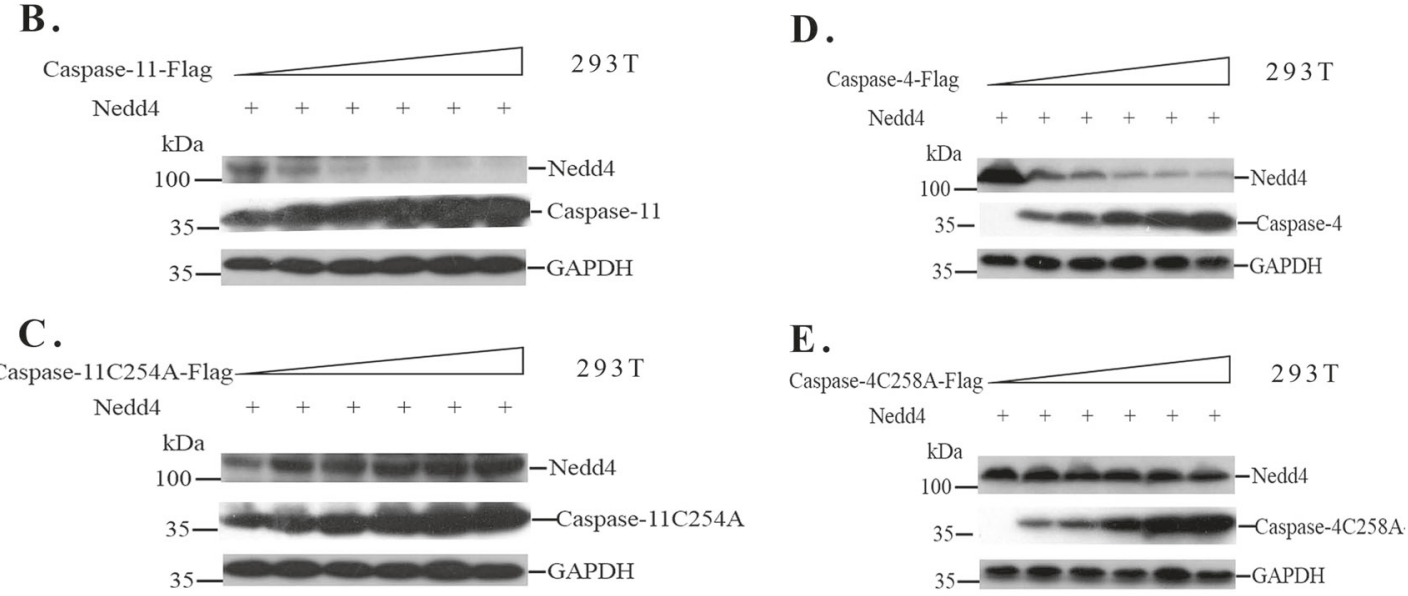

F.

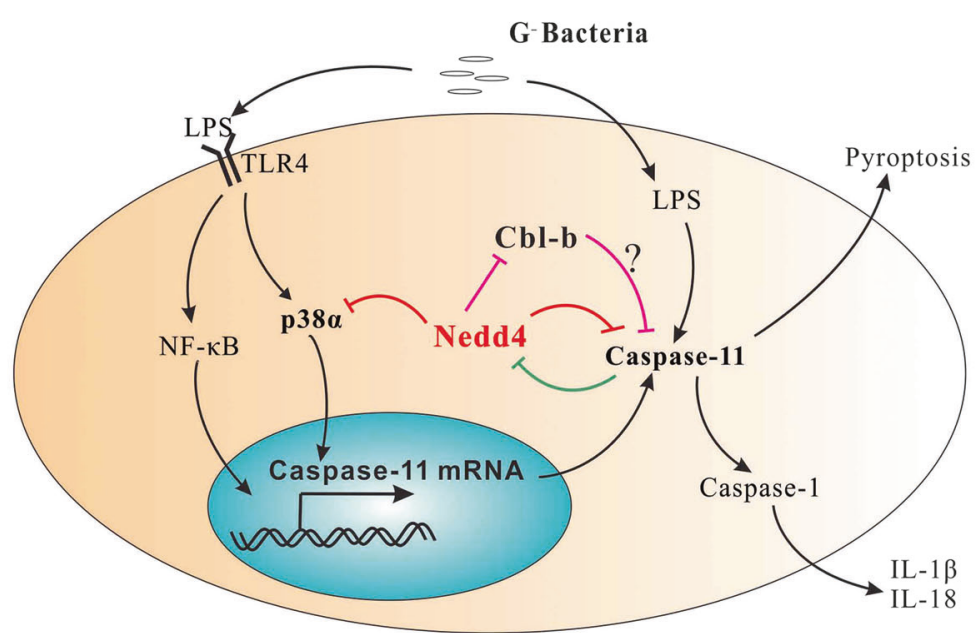

Fig. 7 Nedd 4 can be cleaved by caspase- 11 and caspase-4. a Caspase11 induced by LPS decreases the levels of Nedd4 protein in iBMDM cells. b, c. Immunoblot analyses of lysates from HEK293T cells cotransfected with equivalent amounts of mouse Nedd4 plus increasing amounts of caspase-11 (b) or caspase-11C254A (c), or with equivalent amounts ofhuman Nedd4 plus increasing amounts of caspase-4 (d) or caspase-4C258A (e). f Model of mutual regulation between Nedd4 and the non-canonical inflammasome. LPS from gram-negative bacteria induces caspase-11 mRNA expression through the TLR4 pathway, which includes NF-kB and p38a. Meanwhile, cytosolic LPS activates

\section{Plasmids and transfection}

pcDNA3.1(-)-HA-Nedd4, pcDNA3.1(-)-HA-Nedd4C744E, pcDNA3.1(-)-HA-hNedd4, pcDNA3.1-Ub-HA, pcDNA3.1- caspase-11, which leads to pyroptosis and activation of caspase-1 to release IL-18 and IL- $1 \beta$. The inhibitory effect of Nedd4 on noncanonical inflammasome activation might be mediated by the ubiquitination of $\mathrm{p} 38 \mathrm{a}$ and caspase-11, and their subsequent degradation. Conversely, caspase- 11 cleaves Nedd4 to remove its inhibitory effect. Cbl-b inhitits non-canonical inflammasome activation by unconfirmed mechanism. However, Nedd4 is responsible for Cbl-b ubiquitination and its degradation, thus, Nedd4 inhibits non-canonical inflammasome activation independent on Cbl-b

UbK48-HA and pcDNA3.1-UbK63-HA have been previously described [27]. pCS2-caspase-11, pCS2-caspase11C254A, pCS2-caspase-4, pCS2-caspase-4C258A, pCS2caspase-11CARD and pCS2-caspase-11 $\triangle \mathrm{CARD}$ were gifts 
from Professor Feng Shao (NIBS), and have been previously described [7]. HEK293T cells were transfected with the indicated expression plasmids using Lipofectamine 3000. The cells were then lysed in different lysis buffers, as required for subsequent experiments.

\section{ELISA}

IL-1 $\beta$, IL-6, and TNF- $\alpha$ in mouse serum were measured by ELISA according to the manufacturer's manual. Assays were performed in triplicate for each independent experiment.

\section{Cytotoxicity assay}

Cytotoxicity was measured with a Cytotoxicity Detection Kit (LDH) according to the manufacturer's manual. Assays were performed in triplicate for each independent experiment.

\section{Immunoblotting}

For protein interaction assays, cells were lysed with $0.5 \%$ NP-40 buffer. For ubiquitination assays, cells were lysed in ice-cold radioimmunoprecipitation assay (RIPA) lysis buffer containing $50 \mathrm{mM}$ Tris- $\mathrm{HCl}, \mathrm{pH} 8.0,150 \mathrm{mM} \mathrm{NaCl}, 1 \%$ Nonidet P-40, $0.5 \%$ deoxycholate, $0.1 \%$ SDS, and a protease inhibitor cocktail. After centrifugation, cell extracts were resolved by SDS-PAGE and analyzed by immunoblotting. The membranes were probed with the indicated antibodies. Blots were visualized by Clarity $^{\mathrm{TM}}$ western enhanced chemiluminescence substrate and exposure on X-ray film (Kodak) or Tianneng image system.

\section{Histochemistry}

To assess the lesions of the tissues resulting from noncanonical inflammasome activation, paraffin sections were stained with haematoxylin and eosin. The heart, liver, spleen, lung and kidney were fixed in 4\% formaldehyde solution for $24 \mathrm{~h}$ before being washed with PBS. After dehydration through a graded ethanol series, slices were immersed in a 1:1 mixture of $100 \%$ ethanol and wax for 1 $\mathrm{h}$ at $37^{\circ} \mathrm{C}$, followed by two 1 -h incubations in pure wax at $37^{\circ} \mathrm{C}$ and then embedded in wax and cut into 3-mm-thick slices. Tissue sections were stained with hematoxylin and eosin for the assessment of pathological changes by light microscopy. The pathological changes were observed and scored with 4 grades by a pathologist. In brief, for heart pathology, (1) Normal, (2) The transverse striations of myocardial fibers were clear, (3) The lesions of myocardial fibers were diffused, and (4) The lesions of myocardial fibers were diffused and local necrotic changes are evident. For liver pathology, (1) Normal, (2) The focal necrosis and inflammatory cell infiltration were occasionally seen in liver tissue, (3) The patchy necrosis and large necrosis were seen in liver tissue, and the lesions were limited, and (4) The patchy necrosis and large necrosis were seen in liver tissue, and the lesions were diffused.

To further access expressions of caspase-11 on the liver, immunohistochemical stainings for liver sections prepared as above were done. The Caspase-11 antibody (17D9) (NB120-10454, novus) analysis of paraffin embedded mice livers was at 1:100 dilution. Polink-2 plus ${ }^{\circledR}$ Polymer HRP Detection System (PV-9002) was used to detect mouse primary antibody. The stained slides were loaded onto the Vectra multispectral microscope system (Perkin Elmer, Waltham, MA) and analyzed by Image-Pro Plus 6.0.

\section{Immunoprecipitation}

Cell lysates were prepared in ice-cold lysis buffer, and immunoprecipitated with the indicated antibodies in immunoprecipitation buffer containing $10 \mathrm{mM}$ Tris- $\mathrm{HCl}$, $\mathrm{pH} 7.0,150 \mathrm{mM} \mathrm{NaCl}, 0.5 \% \mathrm{NP}-40$ and protease inhibitors $(1: 500)$ for $4 \mathrm{~h}$ at $4{ }^{\circ} \mathrm{C}$. All samples were incubated with protein $\mathrm{A} / \mathrm{G}-\mathrm{Sepharose}$ overnight at $4{ }^{\circ} \mathrm{C}$. The beads were washed three times with ice-cold lysis buffer or immunoprecipitation buffer, recovered protein was separated by SDS-PAGE and analyzed by immunoblotting with the indicated antibodies. Blots were visualized with chemiluminescence reagent as described above.

\section{In vivo ubiquitination assays}

Twenty-four hours post-transfection, MG132 (10 $\mu \mathrm{M})$ or chloroquine $(40 \mu \mathrm{M})$ were added for $8 \mathrm{~h}$, the cells were lysed with harsh RIPA lysis buffer containing protease inhibitors (1:500) and 1\% SDS, on ice for $1 \mathrm{~h}$, and then denatured by heating for $5 \mathrm{~min}$. After ultrasonicating three times (with a Dr. Hielscher UP200S instrument; 2 min/ time, $0.5 \times 50 \%$ ), the supernatants were diluted with lysis buffer until the concentration of SDS was decreased to $0.1 \%$, and then re-immunoprecipitated with the appropriate antibodies. Immunoblot analysis of the Ni-NTAbinding fraction (pulling down ubiquitin-his) of lysates from HEK293T cells co-transfected with various vectors (Fig. 5a), stained with an anti-Flag antibody. The lysate HEK293T cells transfected with Flag-tagged protein and HA-tagged ubiquitin (Fig. 5b). was finally immunoprecipitated with anti-Flag antibody overnight at $4{ }^{\circ} \mathrm{C}$, and pulled-down with protein A/G beads (Fig. 5b). The samples were washed three times with RIPA lysis buffer, gelseparated and analyzed by immunoblotting with anti-HA antibody. 


\section{Liquid chromatography-MS}

Sample preparation for mass analysis was according to the method [27].

MS data were acquired by Beijing BangFei Bioscience Co., Ltd using a Thermo Q-Exactive mass spectrometer. Samples of $20 \mu \mathrm{l}$ were injected onto a C18 column (C18, $1.9 \mu \mathrm{m}, 0.15 \times 120 \mathrm{~mm})$, and then trapped with another column $(\mathrm{C} 18,3 \mu \mathrm{m}, 0.10 \times 20 \mathrm{~mm})$ using $0.1 \%$ formic acid in water as mobile phase $\mathrm{A}$ and $0.1 \%$ formic acid in $80 \%$ acetonitrile as mobile phase $\mathrm{B}$, at a flow rate of 600 $\mathrm{nl} / \mathrm{min}$. Separation was attained using a solvent gradient ranging from $5 \%$ to $95 \%$ of mobile phase B over $78 \mathrm{~min}$. For the survey full-scan MS mode, the mass scanning range was set from $\mathrm{m} / \mathrm{z}, 300$ to 1400 (resolution was set at 70,000 , automatic gain control target at $3 \times 10^{6}$, maximum at $60 \mathrm{~ms}$ ). For data dependent-MS [2] /data dependent selected ion monitoring, the following parameters were set: resolution at 17,500, automatic gain control target at $5 \times 10^{4}$, maximum at $80 \mathrm{~ms}$, loop count at 20 , isolation window at $3.0 \mathrm{~m} / \mathrm{z}$, fixed first mass at $100.0 \mathrm{~m} / \mathrm{z}$, and normalized collision energy/stepped Ac at 27. The data dependence settings were: underfill ratio set at $1.0 \%$, charge exclusion at $1,7,8,>8$, peptide match on, exclude isotopes on, and dynamic exclusion at $18.0 \mathrm{~s}$. The.RAW files data were analyzed using Sequest and Proteome Discoverer (Thermo Scientific) software. The search parameters used in Proteome Discoverer included: the peptide mass tolerance was $\pm 15 \mathrm{ppm}$, the fragment mass tolerance was $0.02 \mathrm{Da}$, and the enzyme was assigned as trypsin with two missed cleavages allowed. The variable post-translational modifications in the search parameters were assigned to include the oxidation of methionine, acetylation and ubiquitination of lysine.

\section{Statistical analysis}

Data are expressed as the mean $\pm \mathrm{SD}$. All statistical analyses were conducted using SPSS 18.0 software. Student's t test was used to assess the difference between controls and the indicated groups. Unless indicated, results were derived from three independent experiments with similar results. Error bars on graphs indicate SD.

Acknowledgements We thank Professor Feng Shao (NIBS, China) for the iBMDM cell line and caspase-11/4 expressing vectors. We thank Professor Baoli Yang (Department of Obstetrics and Gynecology, University of Iowa, Iowa City, IA 52242, USA) for supplying Nedd $4^{+/-}$ mice. It was supported by the National Natural Science Foundation of China (81699148).

Author contributions QL conceived and designed the experiments, QL, SZ, ZS and XG performed the experiments, QL analyzed the data and wrote the paper, $\mathrm{HZ}$ guided the overall experimental progress. QL, SZ, ZS were co-first authors.

\section{Compliance with ethical standards}

Conflict of interest The authors declare that they have no conflict of interest.

Publisher's note: Springer Nature remains neutral with regard to jurisdictional claims in published maps and institutional affiliations.

\section{References}

1. Martinon F, Mayor A, Tschopp J. The inflammasomes: guardians of the body. Annu Rev Immunol. 2009;27:229-65.

2. Schroder K, Tschopp J. The inflammasomes. Cell. 2010;140:821-32.

3. Kayagaki N, Warming S, Lamkanfi M, Vande Walle L, Louie S, Dong $\mathrm{J}$, et al. Non-canonical inflammasome activation targets caspase-11. Nature. 2011;479:117-21.

4. Crawford ED, Wells JA. Caspase substrates and cellular remodeling. Annu Rev Biochem. 2011;80:1055-87.

5. von Moltke J, Ayres JS, Kofoed EM, Chavarria-Smith J, Vance RE. Recognition of bacteria by inflammasomes. Annu Rev Immunol. 2013;31:73-106.

6. Hagar JA, Powell DA, Aachoui Y, Ernst RK, Miao EA. Cytoplasmic LPS activates caspase-11: implications in TLR4independent endotoxic shock. Science. 2013;341:1250-3.

7. Shi J, Zhao Y, Wang Y, Gao W, Ding J, Li P, et al. Inflammatory caspases are innate immune receptors for intracellular LPS. Nature. 2014;514:187-92.

8. Kayagaki N, Stowe IB, Lee BL, O'Rourke K, Anderson K, Warming $\mathrm{S}$, et al. Caspase-11 cleaves gasdermin $\mathrm{D}$ for noncanonical inflammasome signaling. Nature. 2015;526:666.

9. Shi J, Zhao Y, Wang K, Shi X, Wang Y, Huang H, et al. Cleavage of GSDMD by inflammatory caspases determines pyroptotic cell death. Nature. 2015;526:660.

10. Aachoui Y, Leaf IA, Hagar JA, Fontana MF, Campos CG, Zak $\mathrm{DE}$, et al. Caspase- 11 protects against bacteria that escape the vacuole. Science. 2013;339:975-8.

11. Broz P, Ruby T, Belhocine K, Bouley DM, Kayagaki N, Dixit VM, et al. Caspase-11 increases susceptibility to Salmonella infection in the absence of caspase-1. Nature. 2012;490:288-91.

12. Gurung P, Malireddi RK, Anand PK, Demon D, Walle LV, Liu Z, et al. Toll or interleukin-1 receptor (TIR) domain-containing adaptor inducing interferon-beta (TRIF)-mediated caspase-11 protease production integrates Toll-like receptor 4 (TLR4) protein- and Nlrp3 inflammasome-mediated host defense against enteropathogens. J Biol Chem. 2012;287:34474-83.

13. Rathinam VA, Vanaja SK, Waggoner L, Sokolovska A, Becker C, Stuart LM, et al. TRIF licenses caspase-11-dependent NLRP3 inflammasome activation by gram-negative bacteria. Cell. 2012;150:606-19.

14. Bhoj VG, Chen ZJ. Ubiquitylation in innate and adaptive immunity. Nature. 2009;458:430-7.

15. Zinngrebe J, Montinaro A, Peltzer N, Walczak H. Ubiquitin in the immune system. EMBO Rep. 2014;15:28-45.

16. Pickart CM. Mechanisms underlying ubiquitination. Annu Rev Biochem. 2001;70:503-33.

17. Hilt W, Wolf DH. The ubiquitin-proteasome system: past, present and future. Cell Mol Life Sci. 2004;61:1545.

18. Hershko A, Ciechanover A. The ubiquitin system. Annu Rev Biochem. 1998;67:425-79.

19. Hicke L, Dunn R. Regulation of membrane protein transport by ubiquitin and ubiquitin-binding proteins. Annu Rev Cell Dev Biol. 2003;19:141-72. 
20. Ingham RJ, Gish G, Pawson T. The Nedd4 family of E3 ubiquitin ligases: functional diversity within a common modular architecture. Oncogene. 2004;23:1972-84.

21. Boase NA, Kumar S. NEDD4: The founding member of a family of ubiquitin-protein ligases. Gene. 2015;557:113-22.

22. Fan CD, Lum MA, Xu C, Black JD, Wang X. Ubiquitindependent regulation of phospho-AKT dynamics by the ubiquitin E3 ligase, NEDD4-1, in the insulin-like growth factor-1 response. J Biol Chem. 2013;288:1674-84.

23. Cao XR, Lill NL, Boase N, Shi PP, Croucher DR, Shan H, et al. Nedd 4 controls animal growth by regulating IGF-1 signaling. Sci Signal. 2008;1:ra5.

24. Wang X, Trotman LC, Koppie T, Alimonti A, Chen Z, Gao Z, et al. NEDD4-1 is a proto-oncogenic ubiquitin ligase for PTEN. Cell. 2007;128:129-39.

25. Yang B, Gay DL, MacLeod MK, Cao X, Hala T, Sweezer EM, et al. Nedd4 augments the adaptive immune response by promoting ubiquitin-mediated degradation of Cbl-b in activated T cells. Nat Immunol. 2008;9:1356-63.

26. Fang DF, He K, Wang N, Sang ZH, Qiu X, Xu G, et al. NEDD4 ubiquitinates TRAF3 to promote CD40-mediated AKT activation. Nat Commun. 2014;5:4513

27. Liu Q, Zhang S, Chen G, Zhou H. E3 ubiquitin ligase Nedd4 inhibits AP-1 activity and TNF- $\alpha$ production through targeting p38 $\alpha$ for polyubiquitination and subsequent degradation. Sci Rep. 2017;7:4521.

28. Liu Q, Guo H, Xiao Y, Zhao Y, Zhang J. E3 ubiquitin ligase Cblb inhibits NLRP3 inflammasome activation by targeting NLRP3 for ubiquitination (INM6P.405). J Immunol. 2014;192(1 Supplement):122/2.
29. Kayagaki N, Wong MT, Stowe IB, Ramani SR, Gonzalez LC, Akashi-Takamura $\mathrm{S}$, et al. Noncanonical inflammasome activation by intracellular LPS independent of TLR4. Science. 2013;341:1246-9.

30. Yang D, He Y, Munoz-Planillo R, Liu Q, Nunez G. Caspase-11 Requires the Pannexin-1 Channel and the Purinergic P2X7 Pore to Mediate Pyroptosis and Endotoxic Shock. Immunity. 2015;43:923-32.

31. Hur J, Kim SY, Kim H, Cha S, Lee MS, Suk K. Induction of caspase-11 by inflammatory stimuli in rat astrocytes: lipopolysaccharide induction through p38 mitogen-activated protein kinase pathway. FEBS Lett. 2001;507:157-62.

32. Harvey KF, Harvey NL, Michael JM, Parasivam G, Waterhouse $\mathrm{N}$, Alnemri ES, et al. Caspase-mediated cleavage of the ubiquitinprotein ligase Nedd4 during apoptosis. J Biol Chem. 1998;273:13524-30.

33. Poreba M, Strozyk A, Salvesen GS, Drag M. Caspase substrates and inhibitors. Cold Spring Harb Perspect Biol. 2013;5:a008680.

34. Guo H, Callaway JB, Ting JP. Inflammasomes: mechanism of action, role in disease, and therapeutics. Nat Med. 2015;21:677-87.

35. Liu J, Qian C, Cao X. Post-translational modification control of innate immunity. Immunity. 2016;45:15-30.

36. Man SM, Karki R, Kanneganti TD. Molecular mechanisms and functions of pyroptosis, inflammatory caspases and inflammasomes in infectious diseases. Immunol Rev. 2017;277:61-75.

37. Lamkanfi M, Dixit VM. Mechanisms and functions of inflammasomes. Cell. 2014;157:1013-22.

38. Cao X. Self-regulation and cross-regulation of pattern-recognition receptor signalling in health and disease. Nat Rev Immunol. $2016 ; 16: 35-50$. 